Comment on "Dielectric tunability of (110) oriented barium strontium titanate epitaxial films on (100) orthorhombic substrates" [Appl. Phys. Lett. 89, 042903 (2006)]

A. G. Zembilgotov, U. Böttger, and R. Waser

Citation: Appl. Phys. Lett. 90, 036101 (2007); doi: 10.1063/1.2430492

View online: https://doi.org/10.1063/1.2430492

View Table of Contents: http://aip.scitation.org/toc/apl/90/3

Published by the American Institute of Physics

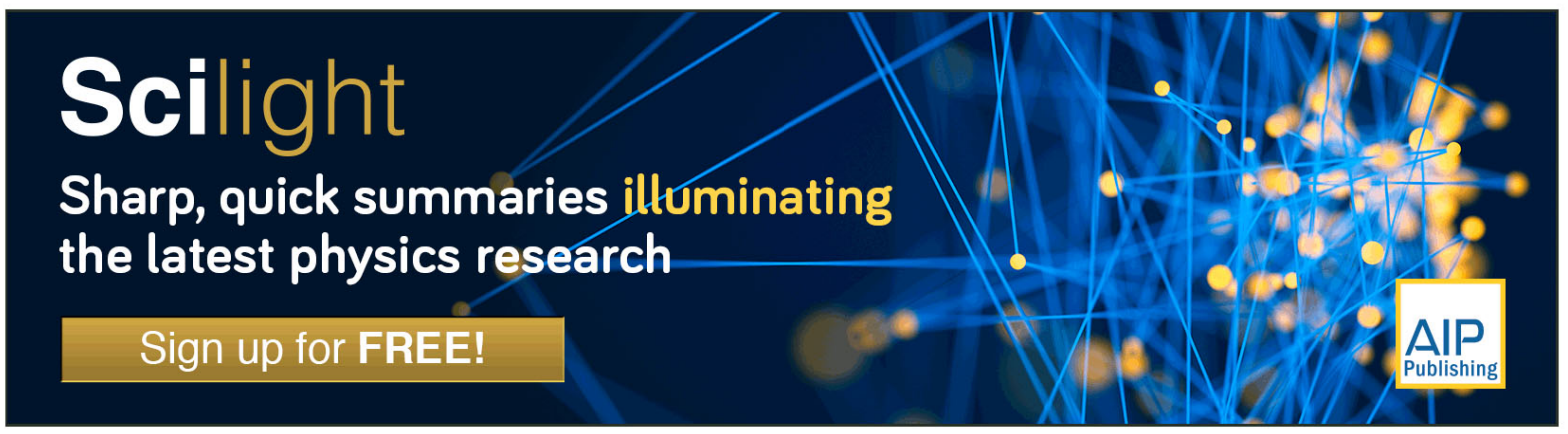




\title{
Comment on "Dielectric tunability of (110) oriented barium strontium titanate epitaxial films on (100) orthorhombic substrates" [Appl. Phys. Lett. 89, 042903 (2006)]
}

\author{
A. G. Zembilgotov, ${ }^{\text {a) }}$ U. Böttger, and R. Waser ${ }^{\text {b) }}$ \\ Institut für Werkstoffe der Elektrotechnik, RWTH Aachen University of Technology, D-52056 Aachen, \\ Germany
}

(Received 24 August 2006; accepted 28 November 2006; published online 18 January 2007)

A modified thermodynamic potential was derived to describe the phase states and dielectric properties of (110) oriented epitaxial films grown on orthorhombic substrates.

[DOI: 10.1063/1.2430492]

In a recent letter Akcay et al. ${ }^{1}$ applied a nonlinear thermodynamic theory to calculate the dielectric properties of (110) oriented ferroelectric films grown on (100) orthorhombic substrates. But there are doubts about the correctness of the thermodynamic potential derived in Ref. 1 for such epitaxial films.

Firstly, the sixth-order terms in this potential [Eq. (4) in Ref. 1] conform to the symmetry of the cubic paraelectric phase. But in the reference frame used by the authors the symmetry of (110) freestanding film differs from the cubic one (Evidently, in this reference frame the symmetry of unconstrained ferroelectric layer is tetragonal). For the discussed case the use of the elastic Gibbs function $G$ in its standard form ${ }^{2}$ is incorrect since this form is not invariant with respect to rotation introduced by the transformation matrix (10) in Ref. 1.

Secondly, the limit of the expressions for renormalized coefficients $\bar{\alpha}_{i}$ for a cubic film with principal axes parallel to the ones of the substrate must coincide with the formulas obtained earlier for (001) oriented film. ${ }^{3}$ [This limit can be easily obtained by settings $C_{11}=C_{22}=C_{33}, C_{12}=C_{13}=C_{23}$, $Q_{11}=Q_{22}, Q_{12}=Q_{13}=Q_{23}$ at the expressions (5) in Ref. 1]. However, the coincidence was not found.

The expression for modified thermodynamic potential $\widetilde{G}$ which correctly takes into account the boundary conditions and the orientation of the film can be obtained by the Legendre transformation ${ }^{4}$ of the Gibbs free energy function rewritten in the film reference frame $\left(x_{1}, x_{2}, x_{3}\right)$. Applying this procedure we obtain the following expression for effective thermodynamic potential of (110) oriented films (the contributions independent of the polarization are omitted):

$$
\begin{gathered}
\widetilde{G}=\alpha_{1}^{*} P_{1}^{2}+\alpha_{2}^{*} P_{2}^{2}+\alpha_{3}^{*} P_{3}^{2}+\Delta \alpha_{11}^{*} P_{1}^{4}+\Delta \alpha_{22}^{*} P_{2}^{4}+\Delta \alpha_{33}^{*} P_{3}^{4} \\
+\Delta \alpha_{12}^{*} P_{1}^{2} P_{2}^{2}+\Delta \alpha_{13}^{*} P_{1}^{2} P_{3}^{2}+\Delta \alpha_{23}^{*} P_{2}^{2} P_{3}^{2}+G_{0}\left(\mathbf{P}^{\prime}\right), \\
\alpha_{1}^{*}=\alpha_{1}+\frac{u_{m 1}\left(Q_{12} s_{12}-Q_{11} s_{22}\right)+u_{m 2}\left(Q_{11} s_{12}-Q_{12} s_{11}\right)}{\left(s_{11} s_{22}-s_{12}^{2}\right)},
\end{gathered}
$$

\footnotetext{
${ }^{a)}$ Permanent address: Institute of Trade and Economics, 194021 St. Petersburg, Russia; electronic mail: az@ domain.ioffe.rssi.ru

b) Also at Institut für Festkörperforschung, Forschungszentrum Jülich, D-52425 Jülich, Germany.
}

$$
\begin{aligned}
& \Delta \alpha_{11}^{*}=\frac{Q_{12}^{2} s_{11}+Q_{11}^{2} s_{22}-2 Q_{11} Q_{12} s_{12}}{2\left(s_{11} s_{22}-s_{12}^{2}\right)}, \\
& \alpha_{2}^{*}=\alpha_{1}+\frac{u_{m 1}\left(Q_{22} s_{12}-Q_{12} s_{22}\right)+u_{m 2}\left(Q_{12} s_{12}-Q_{22} s_{11}\right)}{\left(s_{11} s_{22}-s_{12}^{2}\right)}, \\
& \Delta \alpha_{22}^{*}=\frac{Q_{22}^{2} s_{11}+Q_{12}^{2} s_{22}-2 Q_{12} Q_{22} s_{12}}{2\left(s_{11} s_{22}-s_{12}^{2}\right)}, \\
& \alpha_{3}^{*}=\alpha_{1}+\frac{u_{m 1}\left(Q_{23} s_{12}-Q_{12} s_{22}\right)+u_{m 2}\left(Q_{12} s_{12}-Q_{23} s_{11}\right)}{\left(s_{11} s_{22}-s_{12}^{2}\right)}, \\
& \Delta \alpha_{33}^{*}=\frac{Q_{23}^{2} s_{11}+Q_{12}^{2} s_{22}-2 Q_{12} Q_{23} s_{12}}{2\left(s_{11} s_{22}-s_{12}^{2}\right)}, \\
& \Delta \alpha_{12}^{*}=\frac{Q_{12}\left(Q_{22} s_{11}-Q_{12} s_{12}\right)+Q_{11}\left(Q_{12} s_{22}-Q_{22} s_{12}\right)}{\left(s_{11} s_{22}-s_{12}^{2}\right)} \\
& \Delta \alpha_{13}^{*}=\frac{Q_{12}\left(Q_{23} s_{11}-Q_{12} s_{12}\right)+Q_{11}\left(Q_{12} s_{22}-Q_{23} s_{12}\right)}{\left(s_{11} s_{22}-s_{12}^{2}\right)}, \\
& +\frac{Q_{66}^{2}}{2 s_{66}}, \quad
\end{aligned}
$$$$
\Delta \alpha_{23}^{*}=\frac{Q_{12}\left(Q_{12} s_{22}-Q_{23} s_{12}\right)+Q_{22}\left(Q_{23} s_{11}-Q_{12} s_{12}\right)}{\left(s_{11} s_{22}-s_{12}^{2}\right)},
$$

where $\alpha_{1}$ is the dielectric stiffness coefficient, $s_{l n}$ and $Q_{l n}$ are the elastic compliances at constant polarization and electrostrictive constants, respectively, defined in the film reference frame (Voigt matrix notations is used). The misfit strains $u_{m 1}$ and $u_{m 2}$ can be calculated as $u_{m 1}=\left(a_{1}-a_{0}\right) / a_{0}, \quad u_{m 2}$ $=\left(a_{2}-a_{0}\right) / a_{0}$, where $a_{0}$ is the equivalent cubic cell constant of the freestanding film, $a_{1}$ and $a_{2}$ are the in-plane lattice constants of the epitaxial film. The part of the modified potential $G_{0}\left(\mathbf{P}^{\prime}\right)$ depending only on polarization components can be expressed as 


$$
\begin{aligned}
G_{0}\left(\mathbf{P}^{\prime}\right)= & \alpha_{11}\left(P_{1}^{\prime 4}+P_{2}^{\prime 4}+P_{3}^{\prime 4}\right)+\alpha_{12}\left(P_{1}^{\prime 2} P_{2}^{\prime 2}+P_{1}^{\prime 2} P_{3}^{\prime 2}+P_{2}^{\prime 2} P_{3}^{\prime 2}\right)+\alpha_{111}\left(P_{1}^{\prime 6}+P_{2}^{\prime 6}+P_{3}^{\prime 6}\right)+\alpha_{112}\left[P_{1}^{\prime 2}\left(P_{2}^{\prime 4}+P_{3}^{\prime 4}\right)+P_{2}^{\prime 2}\left(P_{1}^{\prime 4}\right.\right. \\
& \left.\left.+P_{3}^{\prime 4}\right)+P_{3}^{\prime 2}\left(P_{1}^{\prime 4}+P_{2}^{\prime 4}\right)\right]+\alpha_{123} P_{1}^{\prime 2} P_{2}^{\prime 2} P_{3}^{\prime 2}+\alpha_{1111}\left(P_{1}^{\prime 8}+P_{2}^{\prime 8}+P_{3}^{\prime 8}\right)+\alpha_{1122}\left(P_{1}^{\prime 4} P_{2}^{\prime 4}+P_{1}^{\prime 4} P_{3}^{\prime 4}+P_{2}^{\prime 4} P_{3}^{\prime 4}\right) \\
& +\alpha_{1112}\left[P_{1}^{\prime 6}\left(P_{2}^{\prime 2}+P_{3}^{\prime 2}\right)+P_{2}^{\prime 6}\left(P_{1}^{\prime 2}+P_{3}^{\prime 2}\right)+P_{3}^{\prime 6}\left(P_{1}^{\prime 2}+P_{2}^{\prime 2}\right)\right]+\alpha_{1123}\left(P_{1}^{\prime 4} P_{2}^{\prime 2} P_{3}^{\prime 2}+P_{1}^{\prime 2} P_{2}^{\prime 4} P_{3}^{\prime 2}+P_{1}^{\prime 2} P_{2}^{\prime 2} P_{3}^{\prime 4}\right)
\end{aligned}
$$

where $\alpha_{i j}, \alpha_{i j k}$, and $\alpha_{i j k l}$ are the higher order dielectric stiffness coefficients at constant stress, $P_{i}^{\prime}$ are the polarization components in the crystallographic reference frame $\left(x_{1}^{\prime}, x_{2}^{\prime}, x_{3}^{\prime}\right)$ which can be obtained from the ones in the film frame by using the transformation $P_{i}^{\prime}=A_{i j} P_{j}$ with the matrix A given by

$$
\mathbf{A}=\left(\begin{array}{ccc}
1 & 0 & 0 \\
0 & 1 / \sqrt{2} & -1 / \sqrt{2} \\
0 & 1 / \sqrt{2} & 1 / \sqrt{2}
\end{array}\right) .
$$

In its turn, the material constants $Q_{l n}$ and $s_{l n}$ in full tensor notation can be expressed through the ones defined in the crystallographic reference frame as $Q_{\text {mnqp }}$
$=A_{m i}^{-1} A_{n j}^{-1} A_{q k}^{-1} A_{p l}^{-1} Q_{i j k l}^{\prime}, s_{m n q p}=A_{m i}^{-1} A_{n j}^{-1} A_{q k}^{-1} A_{p l}^{-1} s_{i j k l}^{\prime}$, where $\mathbf{A}^{-1}$ is the inverse of $\mathbf{A}$.

The research described in this Comment was made possible in part by Grant 436 RUS 17/71/06 from the Deutsche Forschungsgemeinschaft, Germany.

${ }^{1}$ G. Akcay, I. B. Misirlioglu, and S. P. Alpay, Appl. Phys. Lett. 89, 042903 (2006).

${ }^{2}$ M. J. Haun, E. Furman, S. J. Jang, H. A. McKinstry, and L. E. Cross, J. Appl. Phys. 62, 3331 (1987).

${ }^{3}$ A. G. Zembilgotov, N. A. Pertsev, U. Böttger, and R. Waser, Appl. Phys. Lett. 86, 052903 (2005).

${ }^{4}$ N. A. Pertsev, A. G. Zembilgotov, and A. K. Tagantsev, Phys. Rev. Lett. 80, 1988 (1998). 